\title{
Existência de Controle Ótimo de Sistemas Governados por Equações Integrais de Volterra
}

\author{
Iguer L. D. Santos ${ }^{1}$
}

Departamento de Matemática, Unesp, Ilha Solteira, SP

Resumo. Neste trabalho nós introduzimos uma classe de problemas de controle ótimo de sistemas governados por equações integrais de Volterra. Usando uma propriedade de convergência de soluções para equações integrais de Volterra, nós obtemos a existência de soluções para a classe de problemas de controle ótimo estudada.

Palavras-chave. Controle Ótimo, Equações Integrais de Volterra, Convergência de Soluções

\section{Introdução}

Problemas de controle ótimo de sistemas governados por equações integrais de Volterra foram considerados, por exemplo, em [1], [2], [3] e [4].

Aqui nós estabelecemos a existência de soluções para uma uma classe de problemas de controle ótimo de sistemas governados por equações integrais de Volterra. Para a obtenção da existência de controles ótimos, nós usamos uma propriedade de convergência de soluções similar a [[7], Theorem 3.3].

\section{Preliminares}

Nessa seção consideramos conceitos e resultados utilizados no desenvolvimento do presente trabalho.

\subsection{Equicontinuidade}

Definição 2.1. Seja E uma coleção de funções $f:[a, b] \rightarrow \mathbb{R}^{n}$. Diz-se que E é equicontínuo se para todo $\varepsilon>0$ existe $\delta>0$ tal que

$$
\|f(t)-f(s)\|<\varepsilon
$$

para toda $f \in E$ e para cada $t, s \in[a, b]$ satisfazendo $|t-s|<\delta$.

Abaixo nós enunciamos o Teorema de Arzela-Ascoli.

\footnotetext{
${ }^{1}$ iguerluis@mat.feis.unesp.br
} 
Teorema 2.1 ([6]). Considere uma coleção $E$ de funções $f:[a, b] \rightarrow \mathbb{R}^{n}$. Suponha que E seja equicontínuo e limitado na norma do supremo. Então toda sequência de E possui subsequência que converge uniformemente em $[a, b]$.

A seguir enunciamos a propriedade de continuidade de medida.

Teorema $2.2([6])$. Seja $f:[a, b] \rightarrow[0, \infty)$ uma função em $L_{1}([a, b])$. Dado $\epsilon>0$ existe $\delta>0$ tal que, se $A$ é Lebesgue mensurável e $\mu(A)<\delta$ então

$$
\int_{A} f(s) d s<\epsilon
$$

Observação 2.1. Seja E uma coleção de funções $f:[a, b] \rightarrow \mathbb{R}$. Suponha que exista uma função $\varphi:[a, b] \rightarrow[0, \infty)$ em $L_{1}([a, b])$ tal que

$$
|f(t)-f(s)| \leq \int_{t}^{s} \varphi(\tau) d \tau
$$

para quaisquer $t$ e $s$ satisfazendo $t \leq s$. Do Teorema 2.2 o conjunto E é equicontínuo.

\subsection{Equações integrais de Volterra}

Definição 2.2. Sejam as funções reais $N(t, s)$ e $f:[a, b] \rightarrow \mathbb{R}$, tal que $N$ está definida no plano ts para $t \in[a, b]$ e $s \in[a, t]$. Para $\lambda \in \mathbb{R}$, a equação

$$
x(t)-\lambda \int_{a}^{t} N(t, s) x(s) d s=f(t)
$$

é chamada de equação integral de Volterra de segunda espécie. Dizemos que $N$ é o núcleo desta equação integral.

Teorema 2.3 ([8]). A equação integral de Volterra de segunda espécie

$$
x(t)-\lambda \int_{a}^{t} N(t, s) x(s) d s=f(t)
$$

tal que o núcleo $N$ e a função $f$ são funções contínuas, tem uma única solução contínua. Esta solução é dada pela fórmula

$$
x(t)=f(t)-\lambda \int_{a}^{t} H(t, s ; \lambda) f(s) d s
$$

sendo o núcleo resolvente $H(t, s ; \lambda)$ dado pela série de núcleos iterados

$$
-H(t, s ; \lambda)=\sum_{n=0}^{\infty} \lambda^{n} N_{n+1}(t, s)
$$

tal que

$$
N_{n+1}(t, s)=\int_{s}^{t} N(t, z) N_{n}(z, s) d s \quad n=1,2, \ldots
$$

e $N_{1}(t, s) \equiv N(t, s)$ 
A seguir nós enunciamos e provamos a propriedade de convergência de soluções para equações integrais de Volterra.

Teorema 2.4. Seja $\left\{f_{k}\right\}$ uma sequência equicontínua e uniformemente limitada de funções $f_{k}:[a, b] \rightarrow \mathbb{R}$. Suponha que $\left\|f_{k}-f\right\|_{\infty} \rightarrow 0$. Considere a função contínua $N$ : $[a, b] \times[a, b] \rightarrow \mathbb{R}$. Para cada $k$, seja $x_{k}(t)$ a solução contínua da equação integral

$$
x_{k}(t)=f_{k}(t)+\lambda \int_{a}^{t} N(t, s) x_{k}(s) d s .
$$

Então existe uma subsequência $\left\{x_{k_{j}}\right\} \subset\left\{x_{k}\right\}$ e uma função $x:[a, b] \rightarrow \mathbb{R}$ tal que $\| x_{k_{j}}-$ $x \|_{\infty} \rightarrow 0$, e $x$ satisfaz

$$
x(t)=f(t)+\lambda \int_{a}^{t} N(t, s) x(s) d s .
$$

Demonstração. Sejam $J>0$ e $L>0$ tais que $\left|f_{k}(t)\right| \leq J$ e $|N(t, s)| \leq L$. Temos que

$$
\begin{aligned}
\left|x_{k}(t)\right| & =\left|f_{k}(t)+\lambda \int_{a}^{t} N(t, s) x_{k}(s) d s\right| \leq\left|f_{k}(t)\right|+|\lambda| \int_{a}^{t}\left|N(t, s) x_{k}(s)\right| \Delta s \\
& \leq J+L|\lambda| \int_{a}^{t}\left|x_{k}(s)\right| \Delta s
\end{aligned}
$$

e da desigualdade de Gronwall ([5]) segue que

$$
\left|x_{k}(t)\right| \leq J e^{L|\lambda|(t-a)}
$$

e então

$$
\left|x_{k}(t)\right| \leq J e^{L|\lambda|(b-a)}:=Q
$$

para todo $t \in[a, b]$.

Da continuidade uniforme de $N(t, s)$, para cada $\epsilon>0$, existe $\delta>0$ tal que, se $\left|t-t_{1}\right|<$ $\delta$, então

$$
\left|N(t, s)-N\left(t_{1}, s\right)\right|<\epsilon .
$$

Considere $t$ e $t_{1}$ tais que $\left|t-t_{1}\right|<\delta$ e $t_{1}<t$. Temos

$$
\begin{aligned}
& \left|x_{k}(t)-x_{k}\left(t_{1}\right)\right| \\
& \leq\left|f_{k}(t)-f_{k}\left(t_{1}\right)\right|+|\lambda|\left|\int_{a}^{t_{1}}\left(N(t, s)-N\left(t_{1}, s\right)\right) x_{k}(s) d s\right| \\
& \quad+|\lambda|\left|\int_{t_{1}}^{t} N(t, s) x_{k}(s) d s\right| \\
& \leq\left|f_{k}(t)-f_{k}\left(t_{1}\right)\right|+|\lambda| \epsilon Q(b-a)+|\lambda| L Q\left|t-t_{1}\right| .
\end{aligned}
$$

Esta desigualdade também pode ser verificada para $t<t_{1}$. Como $\left\{f_{k}\right\}$ é uma sequência equicontínua, então $\left\{x_{k}\right\}$ também é uma sequência equicontínua. Do Teorema de ArzelaAscoli existe uma subsequencia de $\left\{x_{k}\right\}$, não reindexamos, tal que $\left\|x_{k}-x\right\|_{\infty} \rightarrow 0$. 
Sendo

$$
x_{k}(t)=f_{k}(t)+\lambda \int_{a}^{t} N(t, s) x_{k}(s) d s
$$

do teorema da convergência dominada ([6]) concluímos que

$$
x(t)=f(t)+\lambda \int_{a}^{t} N(t, s) x(s) d s .
$$

\section{Problemas de controle ótimo de sistemas governados por equações integrais}

Denotamos o conjunto de todas as funções contínuas com domínio $[a, b]$ e contradomínio $\mathbb{R}$ por $C([a, b], \mathbb{R})$. Seja $\varphi:[a, b] \rightarrow[0, \infty)$ uma função em $L_{1}([a, b])$ e seja $\mathcal{U} \subset \mathcal{K}$, onde $\mathcal{K}$ é o conjunto de todas as funções $f:[a, b] \rightarrow \mathbb{R}$ satisfazendo

$$
|f(t)-f(s)| \leq \int_{t}^{s} \varphi(\tau) d \tau
$$

para cada $t$ e $s$ tal que $t \leq s$.

Considere o seguinte problema de controle ótimo $(P)$ :

$$
\min h(x(a), x(b))
$$

sobre todos os pares $(x, u) \in C([a, b], \mathbb{R}) \times \mathcal{U}$, tal que

$$
\left\{\begin{array}{l}
x(t)=u(t)+\lambda \int_{a}^{t} N(t, s) x(s) d s, \quad t \in[a, b] \\
(x(a), x(b)) \in A
\end{array}\right.
$$

onde $A \subset \mathbb{R}^{2}$ é um conjunto fechado e $h: \mathbb{R} \times \mathbb{R} \rightarrow \mathbb{R}$ é uma função semicontínua inferior.

Dizemos que $(x, u) \in C([a, b], \mathbb{R}) \times \mathcal{U}$ é um processo admissível para $(P)$, se o par $(x, u)$ satisfaz a equação $(1)$ e $x$ obedece a condição $(x(a), x(b)) \in A$. Um processo $(\bar{x}, \bar{u})$ é chamado de um processo ótimo para $(P)$, se ele é um processo admissível para $(P)$ que satisfaz

$$
h(\bar{x}(a), \bar{x}(b)) \leq h(x(a), x(b))
$$

para todo processo admissível $(x, u)$ de $(P)$.

Teorema 3.1. Suponha que a função $N:[a, b] \times[a, b] \rightarrow \mathbb{R}$ seja contínua e que o conjunto $\mathcal{U}$ seja limitado na norma do supremo. Então $(P)$ tem um processo ótimo. 
Demonstração. Denote por $\inf \{P\}$ o maior limitante inferior de $h(x(a), x(b))$ sobre todos os processos admissíveis $(x, u)$ de $(P)$. Logo existe uma sequência de processos admissíveis $\left(x_{i}, u_{i}\right)$ de $(P)$ satisfazendo

$$
\inf \{P\}=\lim h\left(x_{i}(a), x_{i}(b)\right)
$$

Como $\left\{u_{i}\right\}$ é uma sequência equicontínua, do Teorema de Arzela-Ascoli existe uma subsequência de $\left\{u_{i}\right\}$, não reindexamos, tal que $\left\|u_{i}-\bar{u}\right\|_{\infty} \rightarrow 0$. Do Teorema 2.4 existe uma subsequência de $\left\{x_{i}\right\}$, não reindexamos, tal que $\left\|x_{i}-\bar{x}\right\|_{\infty} \rightarrow 0$. Além disso, a função $\bar{x}:[a, b] \rightarrow \mathbb{R}$ satisfaz

$$
\bar{x}(t)=\bar{u}(t)+\lambda \int_{a}^{t} N(t, s) \bar{x}(s) d s
$$

em $[a, b]$.

Como $\left(x_{i}(a), x_{i}(b)\right) \in A$ e $A$ é um conjunto fechado, temos que $(\bar{x}(a), \bar{x}(b)) \in A$ e então $(\bar{x}, \bar{u})$ é um processo admissível para $(P)$. Temos também que

$$
\begin{aligned}
\inf \{P\} & =\lim h\left(x_{i}(a), x_{i}(b)\right) \\
& =\liminf h\left(x_{i}(a), x_{i}(b)\right) \\
& \geq h(\bar{x}(a), \bar{x}(b)) \geq \inf \{P\}
\end{aligned}
$$

e portanto $(\bar{x}, \bar{u})$ é um processo ótimo para $(P)$.

\section{Conclusão}

Este trabalho estabeleceu condições suficientes para a existência de controles ótimos para o problema de controle ótimo $(P)$. A existência de soluções para o problema $(P)$ foi obtida no Teorema 3.1 .

\section{Referências}

[1] T. S. Angell, On the optimal control of systems governed by nonlinear Volterra equations, J. Optimization Theory Appl., vol. 19, 29-45, (1976).

[2] S. A. Belbas, Iterative schemes for optimal control of Volterra integral equations, Nonlinear Anal., vol. 37, 57-79, (1999).

[3] S. A. Belbas, A new method for optimal control of Volterra integral equations, Appl. Math. Comput., vol. 189, 1902-1915, (2007).

[4] D. A. Carlson, An elementary proof of the maximum principle for optimal control problems governed by a Volterra integral equation, J. Optim. Theory Appl., vol. 54, 43-61, (1987).

[5] J. K. Hale, Ordinary Differential Equations, Wiley-Interscience, New York, (1969). 
[6] W. Rudin, Real and Complex Analysis, 3rd edn. McGraw-Hill Book Co., New York, (1987).

[7] I. L. D. Santos, On Volterra integral equations on time scales, Mediterr. J. Math., vol. 12, 471-480, (2015).

[8] F. G. Tricomi, Integral Equations, Dover Publications, Inc., New York, (1985). 